\title{
Diseño y análisis estadístico de un instrumento dirigido a evaluar el Trastorno por déficit de atención e hiperactividad en población infanto-juvenil
}

\author{
Teresa Díez Martín ${ }^{1}$, Alejandra Aguilar Latorre ${ }^{2}$, Isabel Laporta Herrero ${ }^{3}$, \\ Patricia Latorre Forcén ${ }^{4}$ y Miguel Pascual Oliver ${ }^{5}$ \\ ${ }^{1}$ Hospital Clínico Universitario Lozano Blesa, Zaragoza (España); \\ ${ }^{2}$ Instituto de Investigación Sanitaria Aragón (España); \\ ${ }^{3}$ Universidad Nacional de Educación a Distancia (España); \\ ${ }^{4}$ Hospital Obispo Polanco, Teruel (España); \\ ${ }^{5}$ Hospital Universitario Miguel Servet, Zaragoza (España)
}

\begin{abstract}
El trastorno por déficit de atención e hiperactividad (TDAH) aparece en los primeros años de la infancia y se caracteriza por la presencia de tres síntomas: déficit de atención, impulsividad e hiperactividad, si bien estos dos últimos síntomas están profundamente entrelazados (APA, 2013). El DSM-V establece la especificación de tres subtipos según la presentación predominante en los últimos seis meses: falta de atención, hiperactividad-impulsividad o combinado. El objetivo de este estudio es el de construir y validar un cuestionario para evaluar el TDAH, explorando los factores que lo componen. El cuestionario diseñado cuenta con 27 ítems divididos en tres factores de acuerdo a la tipología del TDAH. Se han llevado a cabo análisis sobre deseabilidad social y calidad técnica de los ítems, así como análisis exploratorios de las dimensiones del cuestionario. Los resultados se muestran en sintonía con la hipótesis inicial de la existencia de tres factores (actividad motora, actividad atencional e impulsividad), pero con cambios en el orden hipotetizado y una discusión de los mismos factores. Los estudios sobre el TDAH revelan la importancia de utilizar un instrumento riguroso de evaluación acorde a la edad del sujeto y de determinar el contexto de evaluación.
\end{abstract}

Palabras clave: TDAH, cuestionario, validación, factores, infanto-juvenil.

An instrument aimed at evaluating $A D H D$ in children and adolescents. Attention Deficit Hyperactivity Disorder (ADHD) appears in the early years of childhood and is characterized by the presence of three symptoms: attention deficit, impulsivity and hyperactivity, although these last two symptoms are deeply intertwined (APA, 2013). The DSM-V establishes the specification of three subtypes according to the predominant presentation in the last six months: lack of attention, hyperactivity-impulsivity or combined. The objective of this study is to construct and validate a questionnaire to assess ADHD, exploring the factors that make it up. The designed questionnaire has 27 items divided into three factors according to the type of ADHD. An analysis of the social desirability and the technical quality of the items as well as an exploratory analysis of the dimensions of the questionnaire has been conducted. The results are shown to be in tune with the initial hypothesis of the existence of three factors (motor activity, attention activity and impulsivity), but with changes in the hypothesized order and a discussion of the same factors. Studies on ADHD reveal the importance of using a rigorous assessment instrument according to the subject's age and determining the context of evaluation.

Keywords: ADHD, assessment instrument, validation, factors, children and adolescents.

Correspondencia: Teresa Díez Martín. C/ Miguel Servet, $\mathrm{n}^{\circ}$ 174, bloque 4, 3º A. C.P.: 50013. Zaragoza (España). E-mail: teresadiezpsicologa@gmail.com 
El Trastorno por déficit de atención e hiperactividad (TDAH) se ha descrito hace mucho tiempo y ha recibido distintos nombres. En 1918 se hablaba de encefalitis; después en los años 30, de daño cerebral mínimo; en los años 60, de disfunción cerebral mínima (Denckia y Heilman, 1979) y finalmente, en 1980, con la aparición del DSM-III, de Trastorno por déficit de atención con o sin hiperactividad, que es el término que se ha utilizado hasta la actualidad (Comeche y Vallejo, 2012).

Barkley (1997) considera que el Trastorno por déficit de atención e hiperactividad lleva a un déficit en la autorregulación de las emociones, en la interiorización de las instrucciones externas y en la planificación y organización de la conducta, que a su vez, agrava el cuadro clínico.

Suele aparecer en los primeros años de la infancia y se caracteriza por la presencia de tres síntomas fundamentales: el déficit de atención, la impulsividad y la hiperactividad, si bien estos dos últimos síntomas están profundamente entrelazados (APA, 2013).

El DSM-V establece la especificación de tres subtipos: presentación combinada, si se cumple el criterio A1 (inatención) y el criterio A2 (hiperactividadimpulsividad) durante los últimos 6 meses, presentación predominante con falta de atención, si se cumple el criterio A1 (inatención) durante los últimos 6 meses y por último, presentación predominante hiperactividad-impulsividad, si se cumple el criterio A2 (hiperactividad-impulsividad) durante los últimos 6 meses.

Continuando con la tipología de la hiperactividad, el tipo hiperactivo-impulsivo es el más conflictivo, el tipo combinado es el más frecuente, siendo el tipo predominante inatento es el más frecuente en niñas (Pérez, Fernández-Hermida, Fernández-Rodríguez, y Amigo, 2003).

Las tasas de prevalencia de este trastorno oscilan en torno al 5 por 100 de la población infantil (Taylor, 1986) lo que puede suponer que en cada clase suele haber un niño con esta patología. Esta cifra está basada en datos clínicos, pero si los datos provienen de padres y profesores, el porcentaje tiende a aumentar considerablemente, incluso hasta un 20 por 100 de los niños (Stewart, 1975). Se trata de un trastorno más frecuente en hombres que en mujeres, con una ratio de entre 2.5:1 a 5.6:1 (Criado-Álvarez y Romo, 2003). Los problemas asociados a este trastorno suelen ser frecuentes y variados, presentando trastornos de conducta, dificultades de aprendizaje junto con bajo rendimiento académico y problemas emocionales o afectivos como depresión, baja autoestima, ansiedad y alteraciones psicosomáticas (House, 2003).

La hipótesis de dicha investigación propone que el trastorno de hiperactividad estaría compuesto por el factor actividad motora que puede reducirse a intranquilidad o sentimiento interno de nerviosismo o inquietud con el paso de los años (niñez y adolescencia), el factor actividad atencional, pueden afectar al trabajo de clase y a la 
actuación académica, y el factor impulsividad puede llegar a romper con reglas familiares, interpersonales y/o educativas.

El objetivo es comprobar esta hipótesis, a la vez que se analiza la fiabilidad y la validez del cuestionario diseñado y aplicado a los sujetos.

\section{MÉTODO}

\section{Participantes}

La muestra de esta investigación está compuesta por 314 sujetos a los que se les pide cumplimentar el cuestionario de forma anónima y sincera. Se aclara que no hay respuestas correctas o incorrectas ni preguntas con trampa. Además, también se aconseja no dedicar demasiado tiempo en contestar a las preguntas, ya que las primeras reacciones suelen ser las más precisas. Por último, cabe destacar que los ítems son formulados de manera adaptada a la edad de la muestra.

La edad de los sujetos se encuentra en un intervalo que va desde los siete años hasta los quince años. En cuanto a la variable sexo, el 58\% son hombres y el $41.2 \%$ mujeres. Los participantes son elegidos aleatoriamente, en dos colegios de la provincia de Zaragoza. El único criterio necesario a la hora de escoger la muestra es que los participantes estén dentro del rango de edad señalado.

\section{Instrumentos}

Para medir la variable hiperactividad, se utiliza un cuestionario diseñado por el equipo de dicho estudio. Éste está compuesto por 27 ítems, los cuales están estructurados en 3 factores. Un factor está formado por 9 ítems, otro por 10 ítems, y el último por 6 ítems. Para finalizar, el cuestionario también lo componen dos ítems de deseabilidad social. Así, el cuestionario está dividido de la siguiente manera:

Actividad Motora: ítems 2, 4, 10, 15, 19, 22, 24, 26, 27.

Actividad Atencional: ítems 1, 5, 6, 9, 13, 14, 18, 21, 23, 25.

Impulsividad: ítems 3, 7, 11, 12, 16, 20.

Deseabilidad Social: ítems 8, 17.

El cuestionario cuenta con una serie de instrucciones para facilitar la compresión de los participantes y se remarcaba el carácter anónimo de éste. Al inicio de cuestionario hay un apartado de datos personales sobre la edad, el sexo, curso escolar y si ha repetido algún curso el alumno.

La escala que se aplica al cuestionario tiene como puntuación mínima 1, y como máxima 5. El valor 1 corresponde con "Nunca", y el 5 con "Siempre". El resto de las puntuaciones siguen la siguiente escala: 2 "Casi nunca", 3 "Algunas veces", 4 "Casi siempre" y 5 "Siempre". 


\section{Procedimiento}

Previamente a la cumplimentación del cuestionario, se tiene que pedir cita con el director de ambos colegios, informándole acerca de objeto de la investigación, así como de los procedimientos utilizados y garantizando la máxima confidencialidad. Además, el colegio tiene que informar a los padres de los alumnos sobre la investigación y éstos tienen que firmar una autorización, debido a que los sujetos son menores de edad. Se tratan de alumnos desde segundo curso de primaria hasta cuarto curso de educación secundaria obligatoria. El equipo se desplaza hasta el centro para poder explicar a los alumnos las instrucciones verbalmente, aclarar posibles dudas e informar sobre el objetivo del estudio.

\section{Análisis estadísticos}

Primero se lleva a cabo el análisis de fiabilidad (alfa de Cronbach) y se eliminan los ítems con una baja correlación. Se desinvierten los ítems invertidos y se excluyen los sujetos con una puntuación alta en la escala de deseabilidad social. Se evalúa la asimetría y curtosis de los ítems y se suprimen los que no entran en los límites. Finalmente, se realiza un Análisis Factorial Exploratorio para analizar la estructura interna del cuestionario.

\section{RESULTADOS}

Se realiza una serie de análisis para valorar el grado de fiabilidad y validez que tiene el cuestionario. Los ítems 10, 13, 16 y 26 están invertidos y son desinvertidos al realizar los análisis estadísticos (10D, 13D, 16D y 26D).

El primer análisis realizado fue el de fiabilidad. La medida de la fiabilidad mediante el alfa de Cronbach asume que los ítems (medidos en escala tipo Likert) miden un mismo constructo y que están altamente correlacionados (Welch y Comer, 1988). Huh, Delorme, y Reid (2006) establecen que el valor de fiabilidad en investigación exploratoria debe ser igual o mayor a .6; y en estudios confirmatorios entre .7 y .8.

En su primera realización, se obtiene un alfa de Cronbach de .71. Su valor está por encima de .6 por lo tanto es una puntuación fiable, sin embargo, hay una serie de ítems cuya correlación es baja (menor a .25) por lo que se opta por eliminar esos ítems para que la fiabilidad o precisión aumente. Estos 6 ítems (ítems 3, 18, 23, 27, 10D y 16D) son eliminados, por lo tanto, el cuestionario queda con 19 ítems ( social, ítem 8 e ítem 17) y una fiabilidad aumentada, alfa de Cronbach .82.

A continuación, se evalúa la asimetría y curtosis de los ítems seleccionados tras el análisis de fiabilidad. La aproximación a la normalidad que se exige a las variables es mayor o menor dependiendo del autor. Forero et al. (2009) recomienda las distribuciones con coeficientes de asimetría y curtosis en el rango $(-1.5,1.5)$. Los ítems que superan el rango $(-1.5,1.5)$ y se eliminan son los siguientes: $1,4,9,15,20,21$ y 24. 
Posteriormente, se lleva a cabo el análisis de la estructura del cuestionario, con los 12 ítems restantes, mediante un Análisis Factorial Exploratorio. En este análisis se eliminan los sujetos con una puntuación extrema en los ítems de deseabilidad social (=5).

La validez de los resultados de un Análisis Factorial Exploratorio viene condicionada por obtener valores significativos o próximos a la unidad del índice de "adecuación muestral" de Kaiser-Meyer-Olkin (KMO) y el test de esfericidad de Bartlett $(p<.05)$ que se obtienen de la matriz de correlaciones.

En este caso, ambos valores son favorables (KMO=.786; Bartlett, $p<.000) \mathrm{y}$, por tanto, hacen plausible y factible la aplicación del Análisis Factorial Exploratorio al presente cuestionario (Salvador y Gargallo, 2006).

Este análisis se lleva a cabo mediante el método Oblimin directo, método de rotación oblicua, ya que se asume que los factores resultantes guardan un mínimo de relación entre ellos (ver tabla 1 ).

Tabla 1. Varianza total explicada

\begin{tabular}{ccccccc}
\hline \multirow{2}{*}{ Factor } & \multicolumn{5}{c}{ Autovalores iniciales } & \multicolumn{3}{c}{$\begin{array}{c}\text { Sumas extracción de cargas al } \\
\text { cuadrado }\end{array}$} \\
\cline { 2 - 7 } & Total & \% de varianza & \% acumulado & Total & \% de varianza & \% acumulado \\
\hline 1 & 3.228 & 26.899 & 26.899 & 2.582 & 21.520 & 21.520 \\
\hline 2 & 1.429 & 11.911 & 38.810 & .779 & 6.490 & 28.010 \\
\hline 3 & 1.127 & 9.394 & 48.205 & .554 & 4.613 & $\mathbf{3 2 . 6 2 3}$ \\
\hline 4 & .943 & 7.859 & 56.064 & & & \\
\hline 5 & .931 & 7.756 & 63.820 & & \\
\hline 6 & .819 & 6.822 & 70.641 & & \\
\hline 7 & .714 & 5.948 & 76.589 & & \\
\hline 8 & .638 & 5.319 & 81.908 & & \\
\hline 9 & .599 & 4.989 & 86.896 & & \\
\hline 10 & .586 & 4.880 & 91.776 & & \\
\hline 11 & .513 & 4.276 & 96.052 & \\
\hline 12 & .474 & 3.948 & 100.000 & \\
\hline Nota. Método de extracción: factorización de eje principal.
\end{tabular}

En la tabla 1 podemos ver la existencia de tres factores, los cuales en su conjunto explicarían el $32.62 \%$ del total de la varianza.

Al analizar la estructura factorial resultante (ver tabla 2), se observa que la distribución de los ítems en los tres factores no coincide totalmente con la hipotetizada previamente.

Por consiguiente, el cuestionario quedaría constituido de la siguiente manera:

Actividad Motora: ítems 5, 6, 14, 19, 22 y 26D.

Actividad Atencional: ítems 2, 13D y 25.

Impulsividad: ítems 7, 11 y 12. 
Tabla 2. Matriz de patrón

\begin{tabular}{|c|c|c|c|}
\hline & \multicolumn{3}{|c|}{ Factor } \\
\hline & 1 & 2 & 3 \\
\hline 22. Corro y salto en situaciones inapropiadas. & .596 & .102 & -.069 \\
\hline 14. Evito actividades que requieren mucha atención y concentración. & .566 & .131 & .026 \\
\hline $\begin{array}{c}\text { 6. Juego con un lápiz, balanceo la pierna, o hago cualquier otra cosa para relajar los } \\
\text { nervios. }\end{array}$ & .552 & -.045 & -.036 \\
\hline 5. Me distraigo fácilmente con cualquier cosa. & .508 & -.073 & .233 \\
\hline 19. Tiendo a decir y hacer cosas sin pensar. & .361 & .195 & .251 \\
\hline 26D. Cuando empiezo a hacer un trabajo me levanto de la silla & .254 & -.139 & .188 \\
\hline 11. En clase respondo a preguntas antes de que la profesora las termine de formular. & -.050 & .680 & -.017 \\
\hline 7. Interrumpo en clase porque tengo dificultad en realizar actividades en silencio. & .202 & .490 & .088 \\
\hline 12. Interrumpo en actividades o conversaciones de otras personas. & .218 & .316 & .120 \\
\hline 2. Empiezo una actividad sin haber terminado la anterior. & -.172 & .148 & .826 \\
\hline 13D. Cuando empiezo una tarea no la termino & .109 & .052 & .461 \\
\hline 25. Tengo que hacer memoria para saber dónde he dejado las cosas. & .079 & -.130 & .254 \\
\hline
\end{tabular}

Nota. Método de extracción: factorización de eje principal. Método de rotación: Oblimin con normalización Kaiser. D: ítem desinvertido.

\section{DISCUSIÓN Y CONCLUSIÓN}

Uno de los objetivos que se plantea a la hora de realizar esta investigación es comprobar si el test creado con la intención de analizar los distintos niveles de hiperactividad sostenidos bajo tres factores Actividad Motora, Actividad Atencional e Impulsividad era fiable y válido.

En relación con el análisis de fiabilidad los resultados obtenidos avalan la hipótesis propuesta, ya que el valor del Alfa de Cronbach es de .82, pudiéndose concluir que el cuestionario es consistente a nivel interno.

Se debe tener en cuenta que ha habido que eliminar ítems por baja correlación y por superar los límites de asimetría y curtosis. Todo esto puede ser debido, entre otros factores, a una mala concentración de los sujetos, a responder con demasiada rapidez o una lectura poco eficiente de los ítems.

En cuanto a la estructura del cuestionario, los resultados del Análisis Factorial Exploratorio concuerdan con la hipótesis de la existencia de tres factores (APA, 2013). Sin embargo, la saturación de cada ítem en cada factor varía, quedando el factor de actividad motora compuesto por 6 ítems, el de actividad atencional por 3 y el de impulsividad por 3. Este asunto podría mejorarse reconstruyendo el cuestionario, añadiendo nuevos ítems, prestando mayor atención al conjunto de ítems, con el fin de que todos los componentes que conforman el constructo de trastorno por déficit de atención e hiperactividad estuviesen bien representados.

En el desarrollo de esta investigación, quedan algunas cuestiones sin resolver, pues el constructo hiperactividad se puede estudiar y analizar desde distintas posiciones y enfoques. Concretamente, en el estudio realizado, preguntas relacionadas con la edad, el sexo, el nivel de estudios y la repetición o no de un curso escolar han quedado pendientes para futuros análisis. Sería recomendable el reajuste del cuestionario y obtener una 
muestra más amplia, replicar los análisis estadísticos y verificar la estabilidad temporal de los resultados. Se recalca la importancia de realizar un cuestionario breve y de rápida aplicación, validado en el entorno español, con el fin de que funcione como cribado para detectar posibles casos de TDAH en el aula.

\section{REFERENCIAS}

American Psychiatric Association. (1980). Diagnostic and Statistical Manual of Mental Disorders (3. ${ }^{\text {a }}$ ed.) (DSM-III). Wasinghton, DC: APA.

American Psychiatric Association. (2014). Diagnostic and Statistical Manual of Mental Disorders $(D S M-V)$. Wasinghton (trad. cast.: DSM-V. Manual de Diagnóstico y Estadística de los Trastornos Mentales. Panamericana: Madrid, 2013).

Barkley, R.A. (1997). Behavioural inhibition, sustained attention, and executive functions: constructing a unifying theory of ADHD. Psychological Bulletin, 121, 65-94.

Comeche, M.I., y Vallejo, M.A. (2012). Manual de Terapia de Conducta en la Infancia (2 ${ }^{\mathrm{a}}$ ed.). Madrid: Dykinson. I.

Criado-Álvarez J.J., y Romo, B.C. (2003). Variability and tendencies in the consumption of methylphenidate in Spain. An estimation of the prevalence of attention deficit hyperactivity disorder. Revista Neurolología, 37(9), 806-810.

Denckia, M.B., y Heilman, K.M. (1979). The syndrome oh hyperactivity. En K.M. Heilman y E. Valenstein (Eds.), Clinical neuropsychology (pp. 574-597). Nueva York: Oxford University Press.

Forero, C.G., Maydeu-Olivares, A., y Gallardo-Pujol, D. (2009). Factor analysis with ordinal indicators: A Monte Carlo study comparing DWLS and ULS estimation. Structural Equation Modeling, 16(4), 625-641.

House, A.E. (2003). El DSM IV. El diagnóstico en la edad escolar. Madrid: Alianza Editorial.

Huh, J., Delorme, D.E., y Reid L.N. (2006). Perceived third-person effects and consumer attitudes on preventing and banning DTC advertising. Journal of Consumer Affairs, 40(1), 90.

Pérez, A., Fernández, J., Fernádez, C., y Amigo, I. (2003). Guía de tratamientos psicológicos eficaces III. Infancia y adolescencia. Madrid, España: Pirámide.

Salvador, M., y Gargallo, P. (2006). Análisis Factorial [enlínea]. 5campus.com, Estadística. Recuperado el de: http://www.5campus.com/leccion/factorial.

Stewart, M.A. (1975). Niños hiperactivos. En Selecciones de Scientific American: Psicología Contemporánea (pp. 113-118). Madrid: Blume.

Taylor, E.A. (1986). The causes and development of hyperactive behavior. En Taylor EA (Ed.), The overactive child. Clinics in developmental medicine (pp. 188-60). Oxford: Blackwell.

Welch, S., y Comer, J. (1988). Quantitative Methods for Public Administration: Techniques and Applications. USA: Editorial Books/Cole Publishing Co.

Recibido: 8 de julio de 2018

Recepción Modificaciones: 3 de septiembre de 2018

Aceptado: 6 de noviembre de 2018 